\title{
Theories on the On-board Lithium-Ion Battery Electric Power Generator for Infinite Cruising Range Electric Vehicles
}

\section{Katsutoshi Ono*}

Department of Energy Science and Technology, Kyoto University, Tokyo, Japan

\begin{abstract}
There has been considerable interest recently in electric vehicles powered by lithium-ion batteries. To achieve electric vehicles with an infinite cruising range, a new method of charging the lithium-ion battery has been developed, which is termed "electrostatic-induction potential-superposed electrolytic charge (ESI-PSE)". In ESI-PSE, the charge can be completed with power that $12 \%$ of power required for typical direct voltage application. The power generation unit consists of a pair of the same battery modules, in which the performance can be explained through consecutive cycles of alternate charge and discharge between two batteries; when the charge of one battery with the ESI-PSE mode is terminated, it becomes responsible for both the power to recharge the other battery and the power supplied to a motor. This power generator works with zero energy input, zero matter input and zero emission without violating the laws of thermodynamics. Because of simplicity, effectiveness, cleanliness and self-exiting, this specific propulsion system will offer potential route for automobile in the future.
\end{abstract}

Keywords: Electric vehicle; Lithium-ion battery; Infinite cruising range; Electrostatic induction charge; Electrostatic energy; Discharge capacity

\section{Introduction}

Electric vehicles have limited cruising range and also long recharge times. Therefore, to produce energetically ultimate electric vehicles, these disadvantages must be eliminated. To achieve electric vehicle with infinite cruising range requires theoretical consideration on the possibilities of a new propulsion system, i.e., a lithium-ion battery charge-discharge reciprocating electric power generator. This type of generator functions with zero energy input, zero matter input and zero emission. The main point of the particular mechanism of this generation system is the principle of a "zero power input" electrostatic field created between the electrodes during the charging stage. This is converted directly into free energy of the Li embedded in the negative electrode during charge, and is subsequently converted into power during discharge. Here, we intend to address:

- The theoretical foundation for the alternative energy supply system to be connected to the battery module during charge.

- The principle and performance of the electrostatic-induction potential superposed charge of the Li-ion battery module.

- The system design concept for the on-board charge-discharge reciprocating electric power generation system.

- Simulation of the Li-ion battery module performance for a regular passenger car to achieve an infinite cruising range.

\section{Fundamentals}

\section{“Zero power input” electrostatic field}

This approach is first to give an overview of the charge process of a lithium-ion battery. Figure 1 illustrates the electrostatic-to-chemical energy conversion reactions during the charge stage.

When electrons are extracted from the positive Li-oxide electrode, the $\mathrm{Li}^{+}$ions leave the electrode/electrolyte interface. They migrate through an organic solvent electrolyte from the positive to the negative electrode, where they are embedded in the graphene sheet (porous carbon) as a result of accepting electrons from a connected metal lead.
Therefore, it may be said that these electrode reactions may broadly be defined as a type of electrolytic process.

The power supply located outside the cell can only provide power to the areas in the conductive leads and electrodes where free electrons can exist. The free electrons cannot enter the electrolyte between the electrodes. The most extreme area to generate power is the electrode/ electrolyte interface where the electrons can react with the ions from the electrolyte. The energy source available for the processes that occur between the electrodes is the electrostatic field established by a constant voltage applied by the power supply located outside the cell. The ions in the electrolyte are transferred to the electrode under the electrostatic field spontaneously created between the electrodes and that exerts coulombic force. For this process the power supply located outside the cell is not required to supply power to the electrodes, but only a static voltage. Power is not used for the ion transfer process in the cell. In

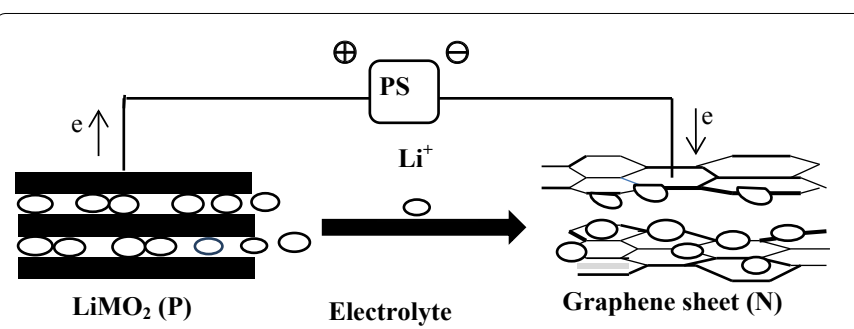

Figure 1: Schematic diagram of the charge process of a lithium-ion battery. PS: Power supply.

*Corresponding author: Katsutoshi Ono, Department of Energy Science and Technology, Kyoto University, Tokyo, Japan, Tel: +8175-753-7531; E-mail: ono6725@tg8.so-net.ne.jp

Received August 28, 2017; Accepted September 11, 2017; Published September 22, 2017

Citation: Ono K (2017) Theories on the On-board Lithium-Ion Battery Electric Power Generator for Infinite Cruising Range Electric Vehicles. J Electr Electron Syst 6: 237. doi: 10.4172/2332-0796.1000237

Copyright: (c) 2017 Ono K. This is an open-access article distributed under the terms of the Creative Commons Attribution License, which permits unrestricted use, distribution, and reproduction in any medium, provided the original author and source are credited. 
addition, many studies [1-4] have formulated the ionic current that flows in the electrostatic field induced by a time-invariable potential difference between electrodes as a function of electrostatic field:

$$
I_{\text {ion }}=\mathrm{Az}^{2} \mathrm{~F}^{2} \mathrm{n} v(-\partial \varphi / \partial x)
$$

where $\mathrm{A}$ is the electrode surface area, $\mathrm{z}$ is the number of electrons, $\mathrm{F}$ is the Faraday constant, $\mathrm{n}$ is the number of moles of ions per unit volume, $v$ is the ion migration velocity, $\varphi$ is the electric potential and $x$ is the direction of migration. This relation indicates that the ionic current flows under the influence of the electrostatic field in the absence of power. Therefore, the electrostatic energy is free of power. This is the principle of "zero power input" electrostatic energy. This energy is then identified as the energy created inside the cell. The original energy that causes the internal energy creation is the intrinsic coulombic force.

\section{Voltage-current characteristics for lithium-ion battery charging}

The charging performance of the Li-ion battery cell can be represented by a nonlinear voltage-current characteristics curve, as shown in Figure $2[5,6]$. This is the generalized V-I curve for the electrochemical decomposition of chemically stable compounds such as $\mathrm{H}_{2} \mathrm{O}$ and $\mathrm{LiMO}_{2}$ (M: transition metal such as $\mathrm{Co}, \mathrm{Ni}, \mathrm{Mn}$ and $\mathrm{Fe}$ ). The voltage at which current begins to flow $(3.7 \mathrm{~V})$ is referred to as the Li extraction potential, $E_{\mathrm{ex}}$, or the $\mathrm{Li}$ redox equilibrium potential for the coexistence of the two-phase, $\mathrm{LiCoO}_{2}+\mathrm{Li}$. This type of electrochemical reaction is generally performed by application of the cell with $E_{\text {ex }}$ plus an extra voltage, $\Delta E$, superimposed on $E_{\mathrm{ex}}$, so the total applied voltage is $E_{\mathrm{ex}}+\Delta E$. The current begins to flow when the voltage is applied over the extraction voltage; the voltage, $E_{\mathrm{ex}}$, is identified as the barrier potential [7]. The external power supply must overcome the effect of the barrier potential before the cell conducts.

The useful "zero power input" electrostatic field exists in the area below the extraction voltage. However, this useful field acts as the barrier potential. When undergoing charge by application of a charge voltage, $E_{\text {ex }}+\Delta E$, and passage of a charge current, $I_{c}$, to a single cell, the power used per cell is given by the relation

$$
P=I_{\mathrm{c}}\left(E_{\mathrm{ex}}+\Delta E\right)=I_{\mathrm{c}} E_{\mathrm{ex}}+I_{\mathrm{c}} \Delta E
$$

The term $I_{c} E_{\text {ex }}$ is the power that the power supply must provide for the current to overcome the barrier potential, and the term $I \Delta E$ is the electrical energy required for the electrode reactions to occur when the charge current flows.

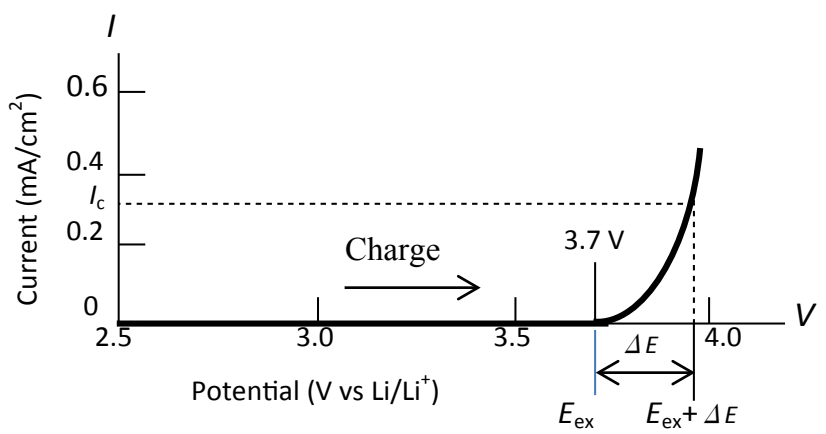

Figure 2: $\mathrm{V}-\mathrm{I}$ curve for the charge process of $\mathrm{Li}$-ion battery with a $\mathrm{LiCoO}_{2}$ positive electrode.

\section{Electrostatic-induction potential-superposed charge of lithium-ion battery}

The power supply method is termed "electrostatic-induction potential-superposed electrolysis (ESI-PSE)”. ESI-PSE consists of a combination of electrostatic induction and electrode potential superposition as shown in Figure 3a. In practice, many cells are assembled into a module configuration. For simplicity a single cell unit is employed to demonstrate the method. The ESI-PSE method supplies potentials to the electrodes in a dual mode, i.e., the superposition of two voltages on the electrodes with two independent voltage sources; one is a bias-voltage source (PS1), which induces $E_{\mathrm{ex}}$ at the cell electrodes and the other is a power supply (PS2), which provides power to the cell. Figure $3 \mathrm{~b}$ shows that the cell electrodes become bipolar.

Figure 4 shows the equivalent closed circuit with two voltage sources connected in opposing directions. These sources can act in parallel independently, and supply the cell electrodes with individual potentials, which yields a superposition such that the resulting voltage between the cell electrodes equals the magnitude of the algebraic sum of the individual potentials $[7,8]$, i.e., $E_{\mathrm{ex}}+\Delta E$. The performance of the cell can be explained by a series of steps. First, when the PS2 output voltage $V_{\mathrm{PS} 2}=E_{\mathrm{ex}} I=0$ (null point) because of the usual condition of uniformity of the electrochemical potential throughout the electrolyte solution [9]. Second, if $V_{\mathrm{PS} 2}$ is increased from the null point, then a charge current $I=I_{\mathrm{c}}$ flows due to the total source voltage (emf) of $\Delta E=V_{\mathrm{PS} 2}-E_{\mathrm{ex}}$. Hence, the two sources can be replaced by a single source that delivers $\Delta E$, according to the superposition theorem of a dc-circuit $[5,6]$. The biasvoltage-source is not required to produce electrical current, but only a static voltage because of the null-current condition at $E_{\mathrm{ex}}$. Therefore, if the ESI-PSE power supply mode is applied, then the total power requirement to charge a single $\mathrm{Li}$-ion battery cell is given by,

$$
P^{\star}=I_{c} \Delta E
$$

The ESI-PSE is a dc electrical circuit that is external to an electrolytic cell, and this is based on the theory of electrostatic induction applied to typical capacitors connected in series and the superposition theorem a

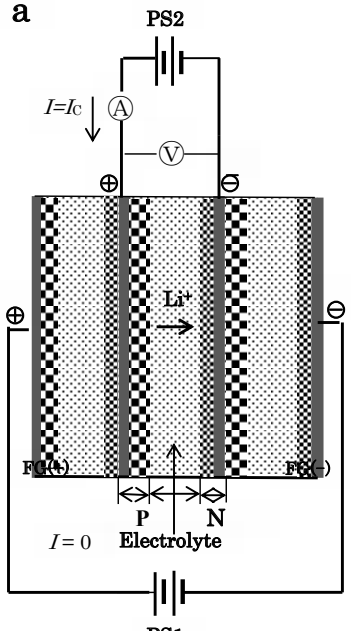

PS1 b

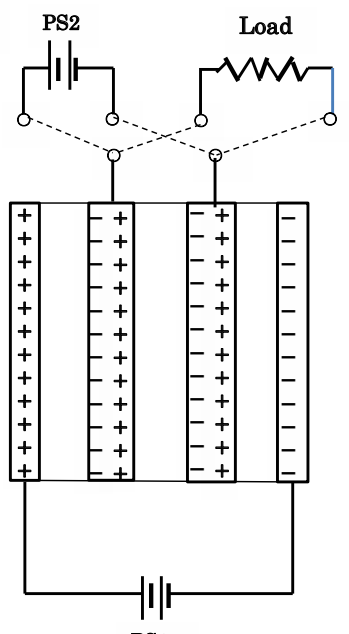

PS1

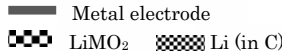

Figure 3: ESI-PSE applied to the charging of a single lithium ion battery cell: (a) Single cell unit. FG: Field generator; P: Positive electrode; N: Negative electrode. (b) Bipolar electrodes during the charge and discharge processes of a Li-ion battery. 


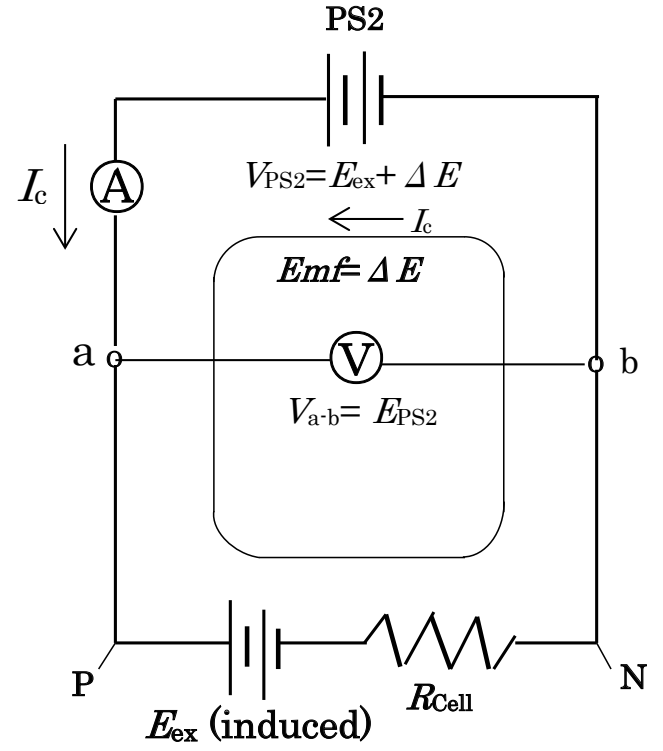

Figure 4: Equivalent circuit for an ESI-PSE lithium ion battery during charge $E_{\mathrm{PS} 2}$ : Output voltage of PS2; $E_{\mathrm{ex}}$ : Induced voltage of the battery cell; /c: Charge current; $\Delta E$ : Extra applied voltage over $E_{\mathrm{ex}} ; V_{\mathrm{a}-\mathrm{b}}$ : Voltage applied between the electrodes $\mathrm{P}$ and $\mathrm{N} ; \mathrm{Rc}_{\mathrm{ell}:}$ Cell resistance.

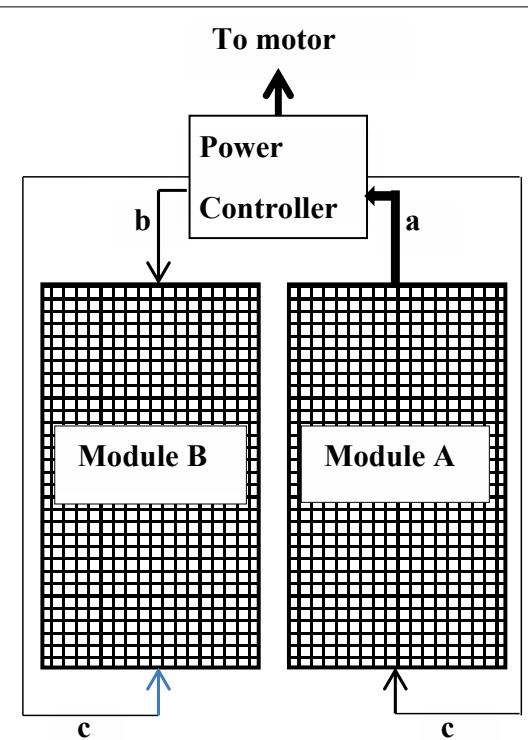

Figure 5: Concept of the charge-discharge reciprocating Li-ion battery electric power generator for infinite cruising range electric vehicles. (a) Power output during discharge; (b): Power recycled for charge with the ESI-PSE power supply mode; (c): Time-invariable voltage applied to the field generators.

of dc circuits. In this sense, every type of electrolytic cell where the $V$-I curve has the same form as in the case of Li-ion battery charging (Figure 2) should give the same form of power requirement as that expressed by eqn. (3). Experimental verification of the ESI-PSE has been performed using a water electrolytic cell and reported previously $[10,11]$.

\section{ESI-PSE power supply mode}

In the ESI-PSE mode, every cell in the module is first adjusted exactly to the time-invariable extraction voltage, $E_{\mathrm{ex}}$, by the electrostatic induction using a bias voltage source PS1 $\left(V_{\mathrm{PS} 1}=3 E_{\mathrm{ex}}\right)$. At this stage, there is no current through the cell. As the second step, power supply PS2 is connected to the electrodes with the polarity opposing the induced voltage $\left(E_{\mathrm{ex}}\right)$. If the voltage of PS2 is the same as $E_{\mathrm{ex}}$, then the current still remains zero. As a next step, if the output voltage of PS2 is set to be $V_{\mathrm{PS} 2}=E_{\mathrm{ex}}+\Delta E$, then a charge current, $I_{\mathrm{c}}$, begins to flow due to the total circuit voltage or the circuit electromotive force, $\Delta E$. Therefore, the power required to charge the cell shown in Figure 3a by the ESI-PSE mode is given by $I_{c} \Delta E$ per cell, according to the eqn. (3) based on the superposition theorem.

Most Li-ion batteries are charged to $4.2 \mathrm{~V}$ per cell. When the battery module is charged in the conventional way with a constant charge voltage of $4.2 \mathrm{~V}$ and a total charge current of $Q(\mathrm{Ah})$, the power used is $4.2 \mathrm{Q}(\mathrm{Wh})$ per cell. On the other hand, when the same module is charged with the ESI-PSE mode, the power used per cell becomes (4.23.7) $Q(\mathrm{Wh})=0.5 \mathrm{Q}(\mathrm{Wh})$, which is only $12 \%$ of that for conventional direct charge voltage applications. The reason for this is that the $\Delta E$ value is particularly small compared to the large $E_{\mathrm{ex}}$-value inherent to the Li-ion battery.

\section{On-Board Lithium-Ion Battery Power Generation System}

The Li-ion battery power generator consists of a couple of identical Li-ion battery modules. The charging of one module is conducted using the discharge capacity of another module. When the discharge is terminated, the situation is changed between these modules. Figure 5 shows a schematic diagram of the charge-discharge reciprocating generation system.

In this case, Module A is in the discharge stage. The field generator voltage is applied continuously during charge and discharge. The opencircuit voltage during discharge is equal to the $\mathrm{Li}$ extraction potential, $E_{\text {ex }}(3.7 \mathrm{~V})$, which is also identical with the equilibrium potential for the coexistence of the two-phases $\mathrm{LiCoO}_{2}+\mathrm{Li}$. Figure 6 shows schematic of the Li-ion battery charge-discharge cycle diagram, i.e., the charge and discharge capacity as function of the electrode potential for a single cell in the module. The battery makes a round of recharge and discharge between the limits, for example, $80 \%$ state of charge (point c) and $80 \%$ depth of discharge (point d). The battery must be put on recharge from the state of point $\mathrm{d}$ (Figure 6) where the electrodes are still bipolar as shown in Figure 3b.

Operation of the charge-discharge reciprocating cycle is described as follows. There is a necessary condition related to the charge time,

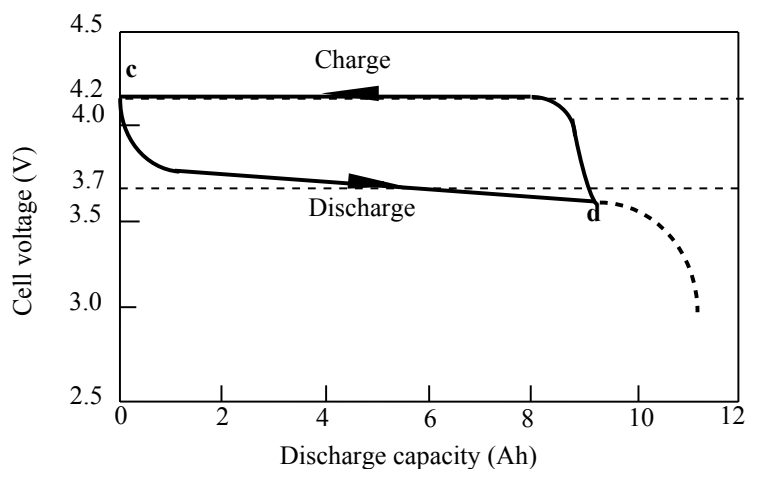

Figure 6: Schematic diagram of the charge-discharge cycle for a single cell of a Li-ion battery module. 
in that one module must complete its charge before the discharge of another module is finished. In this sense, if full power navigation is required, then the user is obliged to drive with a limited range within the charge time. For this evaluation, it is assumed that during a part of the discharge time a vehicle runs with full power, and in the remaining time runs with the official standard cruising (OSC) mode. The energy losses due to degradation of the cell voltage during discharge are neglected. The parameters of concern to estimate the limit of full power navigation are given as below:

$\mathrm{M}_{\mathrm{MAX}}$ : Maximum power of motor; $\mathrm{C}_{\mathrm{B}}$ : Energy capacity of an existing commercial on-board Li-ion battery module; $\mathrm{D}_{\mathrm{C}}$ : Cruising range with the OSC mode; $\mathrm{V}_{\mathrm{AV}}$ : Average cruising velocity with the OSC mode; $\mathrm{R}_{\mathrm{C}}$ : Percentage of the energy capacity of one module to be provided for charging another module; $\mathrm{T}_{\mathrm{C}}$ : Charge time.

The values of each parameter are taken from that of regular commercially available passenger electric vehicle:

$\mathrm{M}_{\mathrm{MAX}}=80 \mathrm{~kW} ; \mathrm{C}_{\mathrm{B}}=45 \mathrm{kWh} ; \mathrm{D}_{\mathrm{C}}=400 \mathrm{~km}$ (Japanese official standard cruising mode, JCO08); $\mathrm{V}_{\mathrm{AV}}=24.4 \mathrm{~km} / \mathrm{h}(\mathrm{JCO} 08) ; \mathrm{R}_{\mathrm{C}}=12 \% ; \mathrm{T}_{\mathrm{C}}=1800 \mathrm{~s}$ (30 min).

This battery module is to be divided into two identical modules; therefore, the energy capacity of module $A$ is $\left(C_{B} / 2\right) 3600[(100$ $\left.\left.R_{C}\right) / 100\right] k W=18 C_{B}\left(100-R_{C}\right)$ at the beginning of discharge. If $T_{F}(s)$ is the time of full power cruising, then the discharge energy by full power cruising is $\mathrm{M}_{\mathrm{MAX}} \mathrm{T}_{\mathrm{F}}$ and that by cruising with the JCO08 mode is $\left(\mathrm{T}_{\mathrm{C}}\right.$ $\left.\mathrm{T}_{\mathrm{F}}\right)\left(\mathrm{C}_{\mathrm{B}} \mathrm{V}_{\mathrm{AV}} / \mathrm{D}_{\mathrm{C}}\right)$. The sum of these values must equal the energy capacity of module $\mathrm{A}$ at the beginning of discharge. From this relationship the value of $\mathrm{T}_{\mathrm{F}}$ is deduced as,

$$
\underset{\mathrm{min}}{\mathrm{T}_{\mathrm{F}}}=\mathrm{C}_{\mathrm{B}}\left[18 \mathrm{D}_{\mathrm{C}}\left(100-\mathrm{R}_{\mathrm{C}}\right)-\mathrm{R}_{\mathrm{C}} \mathrm{V}_{\mathrm{AV}}\right] / \quad\left(\mathrm{D}_{\mathrm{C}} \mathrm{M}_{\mathrm{MAX}}-\mathrm{C}_{\mathrm{B}} \mathrm{V}_{\mathrm{AV}}\right)=858.6 \mathrm{~s}
$$

On the other hand, if a car runs with the JCO08 mode all the time, then the cruising range per discharge is calculated to be $176 \mathrm{~km}$ and the corresponding cruising time is $7.21 \mathrm{~h}$.

\section{Consistency with the Laws of Thermodynamics}

The Li-ion battery power generator functions with zero energy input, zero matter input and zero emission. However, it must satisfy the laws of thermodynamics, so it has no relation to any perpetual motion machine. This Li-ion battery power generation system can be viewed as a closed combined energy cycle of the ESI-PSE module performing the charge process and the identical module performing the discharge process. The sum of "zero power input" electrostatic energy plus the power recycled from the discharge module is converted into the total power output from the discharge module. The total energy delivered by the discharge module equals the total energy required by the charge module; therefore, the "zero power input" electrostatic energy must be equal to the net power output to the outside of the generator. The internal energy created originally by the intrinsic coulombic force is thus recognized as being responsible for the power that can be extracted from the generator. Thus, the overall energy balance does not violate energy conservation.

\section{Applications}

The approach should be applicable to on-board power generator for railway trains and ships, and for stationary type power generators at specific locations.

From an economical perspective, cobalt in the positive electrode is an expensive rare metal. However, ternary or quaternary Li oxides with less expensive transition metals such as $\mathrm{Ni}, \mathrm{Mn}$ and $\mathrm{Fe}$ are now available. These materials will be useful for the present purpose.

\section{Conclusions}

We have investigated an on-board lithium-ion battery electric power generation system that can operate on a mechanism of zero eternal power input from outside the system. The "zero power input" electrostatic energy is converted into the power to drive a motor through two type of direct energy conversions, the electrostatic-tochemical energy conversion during charge and chemical-to-electrical energy conversion during discharge, so that this power generation system does not violate the energy conservation law. From the simplicity, effectiveness, cleanliness and self-exiting of this novel propulsion system, it is expected that it may find practical application to electric vehicles of the future.

\section{References}

1. Bard AJ, Faulkner LR (2001) Electrochemical Methods. John Wiley \& Sons INC.

2. Tamamushi R (1967) Electrochemistry. Tokyo Kagaku Dojin.

3. Newman J, Thomas-Alyea KE (2004) Electrochemical Systems, (3 ${ }^{\text {rd }}$ edn.), Wiley-Interscience, New York.

4. Goodisman J (1987) Electrochemistry, Theoretical Foundations. John Wiley \& Sons, New York.

5. Huang B, Jang YI, Chiang YM, Sadoway DR (2012) Electrochemical evaluation of $\mathrm{LiCoO}_{2}$ synthesized by decomposition and intercalation of hydroxides for lithium-ion battery applications. J Applied Electrochemistry 14: 1365-1369.

6. Ruffo R, Wesselis C, Huggins CR, Cui Y (2009) Electrochemical behavior of $\mathrm{LiCoO}_{2}$ as aqueous lithium-ion battery electrodes. Electrochemistry Communications 11: 247-249.

7. Floyd TL (2004) Electronics Fundamentals. Pearson Education, New Jersey.

8. Satoh T, Sasaki T (2001) Electromagnetics and Electrical Circuit. Nikkan Kogyo Shinbun-sha.

9. Christopher HA, Shipman CW (1968) Poisson's Equation as a Condition of Equilibrium in Electro-chemical Systems. J Electrochem Soc 115: 501-506.

10. Ono K (2015) Energetically Self-Sustaining Electric Power Generation System Based on the Combined Cycle of Electrostatic-Induction Potential-superposed Hydrogen Electrolyzer and Fuel Cell. The Institute of Electrical Engineers of Japan 135: 22-33.

11. Ono K (2016) Hydrogen redox electric power and hydrogen energy generators. International Journal of Hydrogen Energy 41: 10284-10291. 